\title{
Foot posture is associated with kinematics of the foot during gait: A comparison of normal, planus and cavus feet
}

\author{
Andrew K. Buldt ${ }^{\mathrm{a}, \mathrm{b}, *}$, Pazit Levinger ${ }^{\mathrm{c}}$, George S. Murley ${ }^{\mathrm{a}, \mathrm{b}}$, Hylton B. Menz ${ }^{\mathrm{b}}$, \\ Christopher J. Nester ${ }^{\mathrm{d}}$, Karl B. Landorf ${ }^{\mathrm{a}, \mathrm{b}}$ \\ a Discipline of Podiatry, College of Science, Health and Engineering La Trobe University, Bundoora, VIC 3086, Australia \\ ${ }^{\mathrm{b}}$ Lower Extremity and Gait Studies Program, College of Science, Health and Engineering La Trobe University, Bundoora, VIC 3086, Australia \\ ${ }^{\mathrm{C}}$ Institute of Sport, Exercise \&' Active Living, College of Sport and Exercise Science Victoria University, Footscray, VIC 8001, Australia \\ ${ }^{\mathrm{d}}$ School of Health Sciences, University of Salford, UK
}

A R T I C L E I N F O

Article history:

Received 10 December 2014

Received in revised form 2 March 2015

Accepted 4 March 2015

\section{Keywords:}

Foot

Motion

Biomechanics

Walking

Gait

\begin{abstract}
A B S T R A C T
Variations in foot posture are associated with the development of some lower limb injuries. However, the mechanisms underlying this relationship are unclear. The objective of this study was to compare foot kinematics between normal, pes cavus and pes planus foot posture groups using a multi-segment foot model. Ninety-seven healthy adults, aged 18-47 were classified as either normal $(n=37)$, pes cavus $(n=30)$ or pes planus $(n=30)$ based on normative data for the Foot Posture Index, Arch Index and normalised navicular height. A five segment foot model was used to measure tri-planar motion of the rearfoot, midfoot, medial forefoot, lateral forefoot and hallux during barefoot walking at a self-selected speed. Angle at heel contact, peak angle, time to peak angle and range of motion was measured for each segment. One way ANOVAs with post-hoc analyses of mean differences were used to compare foot posture groups. The pes cavus group demonstrated a distinctive pattern of motion compared to the normal and pes planus foot posture groups. Effect sizes of significant mean differences were large and comparable to similar studies. Three key differences in overall foot function were observed between the groups: (i) altered frontal and transverse plane angles of the rearfoot in the pes cavus foot; (ii) Less midfoot motion in the pes cavus foot during initial contact and midstance; and (iii) reduced midfoot frontal plane ROM in the pes planus foot during pre-swing. These findings indicate that foot posture does influence motion of the foot.
\end{abstract}

(c) 2015 Elsevier B.V. All rights reserved.

\section{Introduction}

Prospective studies have found that variations in weightbearing foot posture are associated with an increased risk of medial tibial stress syndrome in military recruits [1] and overuse leg injuries in triathletes [2]. These findings suggest that pes cavus (high medial longitudinal arch) and pes planus (low medial longitudinal arch) may display abnormal biomechanical parameters that predispose an individual to injury. For example, planus feet demonstrate more motion compared to normal feet during gait [3] and as a consequence, may be susceptible to injuries in soft tissues that oppose this motion [4]. In contrast, cavus feet are thought to

\footnotetext{
* Corresponding author at: Discipline of Podiatry, College of Science, Health and Engineering La Trobe University, Bundoora, VIC 3086, Australia. Tel.: +61 394795266.

E-mail address: a.buldt@latrobe.edu.au (A.K. Buldt).
}

exhibit less motion [5] compared to planus feet. Since less energy might be dissipated due to reduced motion, it is hypothesised that such feet are vulnerable to injuries related to impaired shock attenuation [6].

While the mechanisms that link variations in foot posture to injury remain unclear, studies of uninjured participants during walking have found systematic differences in plantar pressure [7] and muscle activity $[8,9]$ in extremes of foot posture (i.e. planus and cavus) compared with normal feet. Thus, external loading and internal forces likely differ between foot types. More ambiguous is the association between foot posture and kinematics. Our recent systematic review [10] found that, compared to the normal feet, planus feet display significantly greater inversion and adduction excursion of the rearfoot (both components of foot supination) during the last $20 \%$ of stance phase (pre-swing). In addition, a positive association was found between pes planus and peak eversion of the rearfoot, which typically occurs in the first $50 \%$ of 
stance. However, our ability to make conclusions was limited due to the absence of a pes cavus group in all except one study. In addition, there was substantial variability in the methods used to classify foot posture, including many that did not have normative data for comparison.

Furthermore, inconsistencies in kinematic methods limit our ability to compare results between studies [10]. For example, one, two and three segment models have been used to represent the foot. Additionally, the protocol for recording static calibration reference trials varied among studies, including placing the participant in a relaxed standing, relaxed seated or a standardised neutral standing position. As such, discrepancies may exist in the zero position from which kinematic data were derived.

The aim of this study was to investigate foot kinematics during gait in people with a normal, cavus or planus foot posture, using validated foot classification methods to classify foot posture and a multi-segment foot modelling technique that adequately addresses the rigid body assumption [11]. Doing so will help us understand the typical motion associated with foot posture and the underlying mechanisms that may be responsible for lower limb overuse injury.

\section{Methods}

\subsection{Participants}

One hundred adults aged 18-47 years were recruited and assigned to one of the three groups based on clinical static foot posture measures. Thirty-seven participants ( 18 males, 19 females) were assigned to the normal group, 32 participants (16 males, 16 females) to the pes planus group, and 31 participants ( 13 males, 18 females) to the pes cavus group. The clinical measures included the 6-item Foot Posture Index (FPI) [12], the Arch Index (AI) [13] and normalised navicular height truncated (NNHt) [14]. To qualify for the normal group, static foot measurement needed to be within one standard deviation of the mean of normative data for the FPI [12], and either the AI or NNHt [15]. Participants were assigned to the pes cavus or pes planus group if static foot measurements were greater or less than one standard deviation of the mean of normative data for the FPI and either the AI or NNHt (Supplementary file).

If only one foot of a participant satisfied selection criteria for a group, then this foot was tested. There was one instance when this occurred and the participant was allocated to the normal group. If both feet qualified, one foot was randomly selected for testing (using the random number generator function in Microsoft Excel ${ }^{\mathbb{R}}$, Microsoft Corporation, Redmond, WA). Participants were free from any current or recurring lower extremity injury that may have affected their ability to walk. Ethical approval was obtained from the La Trobe University Human Ethics Committee (ID number: HEC11-097).

\subsection{Experimental protocol}

Foot kinematics were recorded using ten $100 \mathrm{~Hz}$ Vicon cameras (8 MX2 and 2 MX40, Vicon motion system Ltd., Oxford, England) that detected $9 \mathrm{~mm}$ retro-reflective markers mounted on thermoplastic plates heated and moulded to each participant's foot. A five segment foot model and marker set [16] was used as it has been found to most effectively fulfil the rigid body assumption [11]. Marker locations are displayed in Fig. 1 and documented in Supplementary files.

All participants completed a static calibration trial to define local coordinate frames for each segment and $0^{\circ}$. Participants stood in a relaxed position with the centre of the posterior calcaneus and second toe aligned with the global anterior/posterior axis. After a

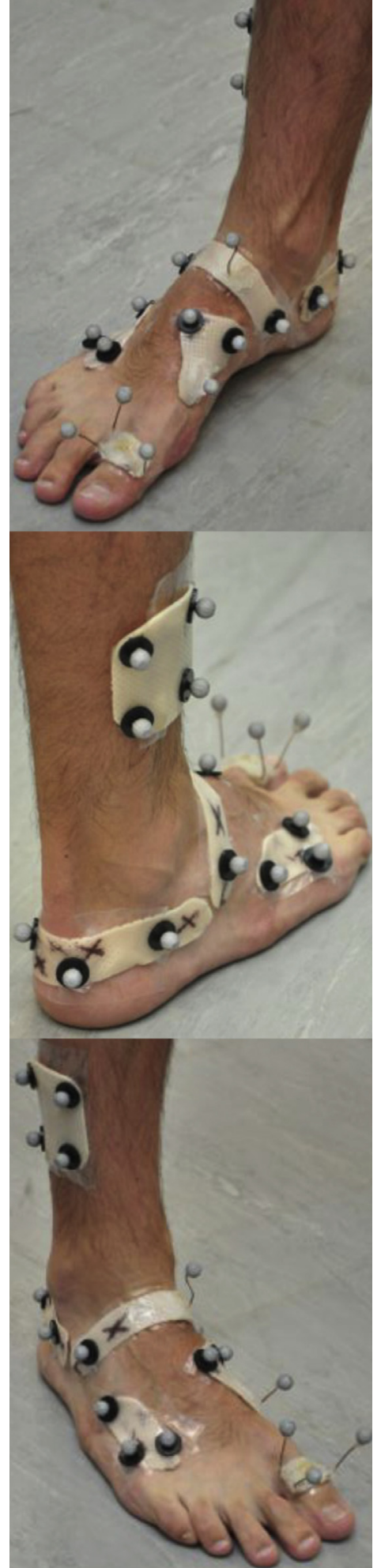

Fig. 1. Marker placement and orientation of the foot for static reference trial.

period of acclimatisation, participants walked at a comfortable pace along a flat $12 \mathrm{~m}$ walkway. A minimum of five acceptable trials were recorded. A trial was deemed acceptable when the participant landed the whole of the tested foot on one force plate (Kistler, type 9865B, Winterthur, Switzerland), and the whole of the contralateral foot on a second force plate (AMTI, OR6, USA) without disrupting gait. 


\subsection{Kinematic evaluation}

Five acceptable trials were processed for each participant. For each trial, heel contact and toe off were determined using vertical ground reaction force data. Walking speed was calculated using force plate data and trials that were within a range $\pm 0.1 \mathrm{~m} / \mathrm{s}$ were processed. The segmental relationships of the foot were as follows:

(i) Rearfoot relative to the lower leg.

(ii) Midfoot relative to the rearfoot.

(iii) Medial forefoot relative to the midfoot.

(iv) Lateral forefoot relative to the midfoot.

(v) Hallux relative to the medial forefoot.

Peak angle of dorsiflexion/plantarflexion, abduction/adduction and inversion/eversion were extracted for all segmental relationships between 0 and $70 \%$ of the gait cycle. This range was used as it captures kinematic variables during stance and early swing phases and ensures that the most relevant peak values for stance phase are recorded. The angle at heel contact was recorded, as was the range of motion (ROM) between the peak values. All angle parameters were expressed as absolute measures relative to the zero position from the static standing trial. Timing of peak angle was expressed as a percentage of the gait cycle.

\subsection{Statistical analysis}

The distribution of data was assessed for skewness, kurtosis and equality of variance (Levene's test). When the assumption of normality was met, a one-way analysis of variance (ANOVA) was performed with significance level set at $<0.05$. When Levene's test indicated significantly different variance between groups and the distribution of the total data set (all groups combined) was normal, one-way ANOVA was performed, with the significance level lowered to 0.01 [17]. Post hoc comparison of the mean differences (MD) between groups with Bonferroni adjustment was applied to all ANOVAs. Confidence intervals (CI) and effect sizes (ES) using Cohen's d were calculated for all significant mean differences.

When Levene's test indicated significantly different variance between groups and the total data set was not normally distributed, a non-parametric test (Kruskal-Wallis test) was performed. All statistical tests were calculated using SPSS version 21 for Windows (IBM Corporation, NY).

\section{Results}

3.1. Participant characteristics

Anthropometric, foot posture classification and spatio-temporal walking measurements are shown in Table 1. The only significant anthropometric difference was in height, with the planus group shorter than both the normal and cavus groups. As there was no related difference in foot length, stride length or walking speed, the difference in height was considered unlikely to cause a difference in foot kinematics and was not included as a covariate. As expected, significant differences were found between all groups for each static foot posture measurements $(p<0.05)$. No significant differences were found in spatio-temporal measurements.

\subsection{Kinematic variables}

Kinematic variables found to have significant differences between the three foot posture groups are presented in Table 2, with all one-way ANOVA results and pairwise comparisons provided in Supplementary files. Graphical representations of the average angular position for all segmental comparisons in all planes are presented in Fig. 2. Comparisons between foot posture groups are described below, and to be concise, only statistically significant results are reported. Taking this into account, there were no significant differences between groups for the medial forefoot relative to the midfoot, so these findings are not reported below. There was one significant non-parametric finding for frontal plane angle of the hallux at heel contact.

Data from two participants in the planus group, one participant in the cavus group and from the midfoot and hallux from two participants in the normal group were excluded due to a technical issue (marker drop out).

3.2.1. Pes cavus compared to normal foot posture

3.2.1.1. Rearfoot relative to the leg. In the transverse plane, the cavus group demonstrated greater abduction at heel contact, less peak adduction (i.e. lesser degree of adduction) during pre-swing (49-60\% of the gait cycle) and less adduction/abduction ROM. In the frontal plane, the cavus group demonstrated greater eversion at heel contact and less peak inversion during pre-swing.

3.2.1.2. Midfoot relative to the rearfoot. In the transverse plane, the cavus group demonstrated greater abduction at heel contact, greater peak abduction during initial contact ( $0-10 \%$ of the gait cycle), and less abduction/adduction ROM during initial contact, midstance and terminal stance (10-48\% of the gait cycle).

3.2.1.3. Lateral forefoot relative to the midfoot. In the transverse plane, the cavus group exhibited greater adduction at heel contact, less peak abduction (i.e. lesser degree of abduction) during midstance and greater peak adduction during preswing.

3.2.1.4. Hallux relative to the medial forefoot. In the frontal plane, the cavus group reached peak eversion earlier and exhibited less peak inversion during pre-swing.

3.2.2. Pes planus compared to normal foot posture

3.2.2.1. Rearfoot relative to the leg. In the transverse plane, the pes planus group demonstrated earlier peak abduction.

Table 1

Participant characteristics. All values are mean (SD) unless otherwise noted.

\begin{tabular}{|c|c|c|c|}
\hline Variable & Normal $(n=37)$ & Pes planus $(n=30)$ & Pes cavus $(n=30)$ \\
\hline \multicolumn{4}{|c|}{ Anthropometric measurements } \\
\hline Gender $(\mathrm{M} / \mathrm{F})-n$ & $18 / 19$ & $15 / 15$ & $13 / 17$ \\
\hline Age (years) & $25.1(4.9)$ & $24.2(5.4)$ & $27.2(7.8)$ \\
\hline Height $(\mathrm{cm})^{*}$ & $173.3(9.4)$ & $166.6(10.1)$ & $174.0(10.3)$ \\
\hline Body mass (kg) & $70.1(13.6)$ & $66.7(14.9)$ & $73.4(14.6)$ \\
\hline Body mass index $\left(\mathrm{kg} / \mathrm{m}^{2}\right)$ & $23.3(3.2)$ & $23.7(3.0)$ & $24.0(2.9)$ \\
\hline Foot length $(\mathrm{mm})$ & $255.8(19.5)$ & $249.4(21.9)$ & $249.9(21.9)$ \\
\hline Left/right foot $-n$ & $20 / 17$ & $15 / 15$ & $17 / 13$ \\
\hline \multicolumn{4}{|l|}{ Clinical measurements } \\
\hline FPI $^{*}$ & 3.9 (1.1) range: $1-6$ & 9.0 (1.0) range: $7-12$ & $-1.41(1.1)$ range: $-3-0$ \\
\hline $\mathrm{AI}^{*}$ & $0.22(0.02)$ range: $0.15-0.29$ & $0.29(0.05)$ range: $0.23-0.40$ & $0.15(0.06)$ range: $0.04-0.24$ \\
\hline $\mathrm{NNHt}^{*}$ & $0.25(0.02)$ range: $0.20-0.32$ & 0.19 (0.03) range: $0.11-0.24$ & $0.32(0.02)$ range: $0.29-0.37$ \\
\hline \multicolumn{4}{|c|}{ Spatio-temporal measurements } \\
\hline Walking speed $(\mathrm{m} / \mathrm{s})$ & $1.35(0.1)$ & $1.32(0.2)$ & $1.33(0.1)$ \\
\hline Stride length (m) & $0.68(0.1)$ & $0.66(0.1)$ & $0.68(0.1)$ \\
\hline
\end{tabular}

FPI, Foot Posture Index; AI, Arch Index; NNHt, normalised navicular height truncated.

Significant difference between groups with one-way ANOVA $(p<0.05)$ 
Table 2

Statistically significant mean differences between foot posture groups with 95\% confidence intervals (CI) and effect sizes (ES). ${ }^{a}$

\begin{tabular}{|c|c|c|c|c|c|c|}
\hline \multirow[t]{2}{*}{ Segmental relationship } & \multirow[t]{2}{*}{ Plane } & \multirow[t]{2}{*}{ Kinematic variable $^{\mathrm{b}}$} & \multicolumn{2}{|c|}{ Mean angle (SD) } & \multirow[t]{2}{*}{ Mean difference $^{c}(\mathrm{CI})^{d}$} & \multirow[t]{2}{*}{ ES } \\
\hline & & & Cavus & Normal & & \\
\hline \multicolumn{7}{|c|}{$\begin{array}{l}\text { Pes cavus compared to normal } \\
\text { Rearfoot/leg }\end{array}$} \\
\hline \multirow{4}{*}{ Rearfoot/leg } & \multirow{2}{*}{ Transverse } & Peak adduction & $-7.1(3.7)$ & $-10.8(3.6)$ & $3.7(1.5$ to 5.9$)$ & 1.01 \\
\hline & & Range of motion & $12.4(2.2)$ & $14.4(2.6)$ & $-1.9(-4.3 \text { to } 0.4)^{\mathrm{e}}$ & 0.83 \\
\hline & \multirow[t]{2}{*}{ Frontal } & Heel contact & $5.1(3.2)$ & $3.2(3.0)$ & $1.9(0.1$ to 3.8$)$ & 0.61 \\
\hline & & Peak inversion & $0.4(2.6)$ & $-1.8(2.9)$ & $2.3(0.6$ to 4.0$)$ & 0.79 \\
\hline \multirow[t]{3}{*}{ Midfoot/rearfoot } & \multirow[t]{3}{*}{ Transverse } & Heel contact & $1.2(1.7)$ & $0.7(1.7)$ & $2.0(0.8$ to 3.1$)$ & 1.10 \\
\hline & & Peak adduction & $0.4(1.7)$ & $-1.8(1.7)$ & $2.2(1.1$ to 3.3$)$ & 1.30 \\
\hline & & Range of motion & $4.2(1.2)$ & $5.5(1.7)$ & $-1.2(-2.2$ to -0.2$)$ & 0.88 \\
\hline \multirow[t]{3}{*}{ Lateral forefoot/midfoot } & \multirow[t]{3}{*}{ Transverse } & Heel contact & $0.7(3.7)$ & $3.9(5.1)$ & $-3.1(-6.1$ to -0.2$)$ & 0.71 \\
\hline & & Peak abduction & $7.3(3.6)$ & $10.6(3.5)$ & $-2.8(-5.5$ to -0.1$)$ & 0.92 \\
\hline & & Peak adduction & $-6.3(4.2)$ & $-2.3(4.0)$ & $-4.0(-6.7$ to -1.3$)$ & 0.97 \\
\hline \multirow[t]{2}{*}{ Hallux/medial forefoot } & \multirow[t]{2}{*}{ Frontal } & Time to peak eversion & $36.7(8.7)$ & $44.9(8.1)$ & $-8.2(-16.0 \text { to }-0.4)^{\mathrm{e}}$ & 0.97 \\
\hline & & Peak inversion & $1.8(8.1)$ & $9.5(11.2)$ & $-7.7(-13.1$ to -2.1$)$ & 0.78 \\
\hline \multirow[t]{2}{*}{ Segmental relationship } & \multirow[t]{2}{*}{ Plane } & \multirow[t]{2}{*}{ Kinematic variable ${ }^{\mathrm{b}}$} & \multicolumn{2}{|c|}{ Mean angle (SD) } & \multirow[t]{2}{*}{ Mean difference $^{c}(\mathrm{CI})^{\mathrm{d}}$} & \multirow[t]{2}{*}{ ES } \\
\hline & & & Planus & Normal & & \\
\hline \multicolumn{6}{|c|}{$\begin{array}{l}\text { Pes planus compared to normal } \\
\text { Rearfoot/leg }\end{array}$} & 0.67 \\
\hline Midfoot/rearfoot & Frontal & Range of motion & $4.5(1.7)$ & $5.9(2.1)$ & $-1.4(-2.6$ to -0.2$)$ & 0.73 \\
\hline Lateral forefoot/midfoot & Transverse & Heel contact & $7.0(5.8)$ & $3.9(5.1)$ & $3.1(0.1$ to 6.0$)$ & 0.56 \\
\hline Hallux/medial forefoot & Sagittal & Peak plantarflexion & $1.6(6.4)$ & $5.4(6.5)$ & $-3.9(-7.5$ to -0.8$)$ & 0.58 \\
\hline \multirow[t]{2}{*}{ Segmental relationship } & Plane & Kinematic variable $^{\mathrm{b}}$ & Mean angle & & Mean difference $^{\mathrm{c}}(\mathrm{CI})^{\mathrm{d}}$ & ES \\
\hline & & & Cavus & Planus & & \\
\hline Pes cavus compared to pe & & & & & & \\
\hline Rearfoot/leg & Transverse & Heel contact & $-0.2(3.3)$ & $-3.3(2.5)$ & $3.1(1.2$ to 5.1$)$ & 1.05 \\
\hline & & Peak abduction & $5.3(3.3)$ & $3.0(2.3)$ & $2.3(0.3 \text { to } 4.2)^{\mathrm{e}}$ & 0.80 \\
\hline & & Time to peak abduction & $28.8(7.3)$ & $21.6(8.2)$ & 7.1 (2.3 to 11.9$)$ & 0.92 \\
\hline & & Peak adduction & $-7.1(3.7)$ & $-12.2(3.5)$ & $5.1(2.8$ to 7.4$)$ & 1.40 \\
\hline & & Range of motion & $12.4(2.2)$ & $15.2(4.2)$ & $-2.8(-5.3 \text { to }-0.4)^{\mathrm{e}}$ & 0.83 \\
\hline & Frontal & Heel contact & $5.1(3.2)$ & $2.4(2.9)$ & $2.7(0.8$ to 4.7$)$ & 0.88 \\
\hline & & Peak inversion & $0.4(2.6)$ & $-2.6(3.1)$ & $3.0(1.2$ to 4.8$)$ & 1.04 \\
\hline Midfoot/rearfoot & Sagittal & Peak dorsiflexion & $1.2(1.9)$ & $2.9(2.4)$ & $-1.6(-3.0$ to -0.3$)$ & 0.78 \\
\hline & Transverse & Heel contact & $1.2(1.7)$ & $-1.2(2.1)$ & $2.5(1.3$ to 3.6$)$ & 1.25 \\
\hline & & Peak adduction & $0.4(1.7)$ & $-1.9(2.0)$ & $2.4(1.2$ to 3.5$)$ & 1.23 \\
\hline & & Range of motion & $4.2(1.2)$ & $6.1(2.0)$ & $-1.8(-2.9$ to -0.8$)$ & 1.15 \\
\hline & Frontal & Range of motion & $6.1(2.2)$ & $4.5(1.7)$ & $1.5(0.2$ to 2.8$)$ & 0.81 \\
\hline Lateral forefoot/midfoot & Transverse & Heel contact & $0.7(3.7)$ & $7.0(5.8)$ & $-6.2(-9.3$ to -3.1$)$ & 1.29 \\
\hline & & Peak abduction & $7.3(6.3)$ & $11.7(5.0)$ & $-4.4(-7.2$ to -1.6$)$ & 0.77 \\
\hline & & Peak adduction & $-6.3(4.2)$ & $-1.0(5.3)$ & $-5.3(-8.2$ to -2.5$)$ & 1.10 \\
\hline & Frontal & Time to peak eversion & $29.9(7.0)$ & $35.3(9.5)$ & $-5.4(-10.6$ to -0.2$)$ & 0.64 \\
\hline & & Time to peak inversion & $60.0(4.8)$ & $62.8(4.2)$ & $-2.8(-5.5$ to -0.1$)$ & 0.62 \\
\hline Hallux/medial forefoot & Frontal & Peak inversion & $1.8(8.1)$ & $9.2(7.1)$ & $-7.3(-13.1$ to -1.6$)$ & 0.95 \\
\hline & & Range of motion & $14.9(7.3)$ & $9.7(5.5)$ & $5.1(1.5$ to 8.8$)$ & 0.80 \\
\hline
\end{tabular}

a Significant result for non-parametric (Kruskal-Wallis) test not included in table.

b Angle values expressed in degrees. Timing values expressed as percentage of gait cycle.

c Mean differences expressed as comparison of absolute angle measures and not an expression of kinematic variables.

d $95 \%$ Confidence intervals unless otherwise signified.

e $99 \%$ Confidence intervals. Significantly different variance between groups but normal distribution of total (groups combined) data set.

3.2.2.2. Midfoot relative to the rearfoot. In the frontal plane, the planus group exhibited less inversion/eversion ROM.

3.2.2.3. Lateral forefoot relative to the midfoot. In the transverse plane, the planus group exhibited greater abduction at heel contact.

3.2.2.4. Hallux relative to the medial forefoot. In the sagittal plane, the planus group exhibited less peak plantarflexion during midstance.

3.2.3. Pes cavus compared to pes planus foot posture

3.2.3.1. Rearfoot relative to the leg. In the transverse plane, the cavus group demonstrated greater abduction at heel contact, later and greater peak abduction during midstance, less peak adduction during pre-swing and less abduction/ adduction ROM. In the frontal plane, the cavus group demonstrated greater eversion at heel contact, and less peak inversion and greater eversion during pre-swing.
3.2.3.2. Midfoot relative to the rearfoot. In the sagittal plane, the cavus group demonstrated less peak dorsiflexion during midstance. In the transverse plane, the cavus group demonstrated greater abduction at heel contact, greater peak abduction during initial contact and less abduction/adduction ROM. In the frontal plane, the cavus group demonstrated greater inversion/eversion ROM.

3.2.3.3. Lateral forefoot relative to the midfoot. In the transverse plane, the cavus group demonstrated greater adduction at heel contact less peak adduction during midstance and greater peak abduction during pre-swing. In the frontal plane, the cavus group reached peak eversion and peak inversion earlier than the pes planus group.

3.2.3.4. Hallux relative to the medial forefoot. In the frontal plane, the cavus group displayed greater peak inversion during pre-swing and greater inversion/eversion ROM. 
Sagittal

Rearfoot relative to the leg

\section{Midfoot relative to} the rearfoot
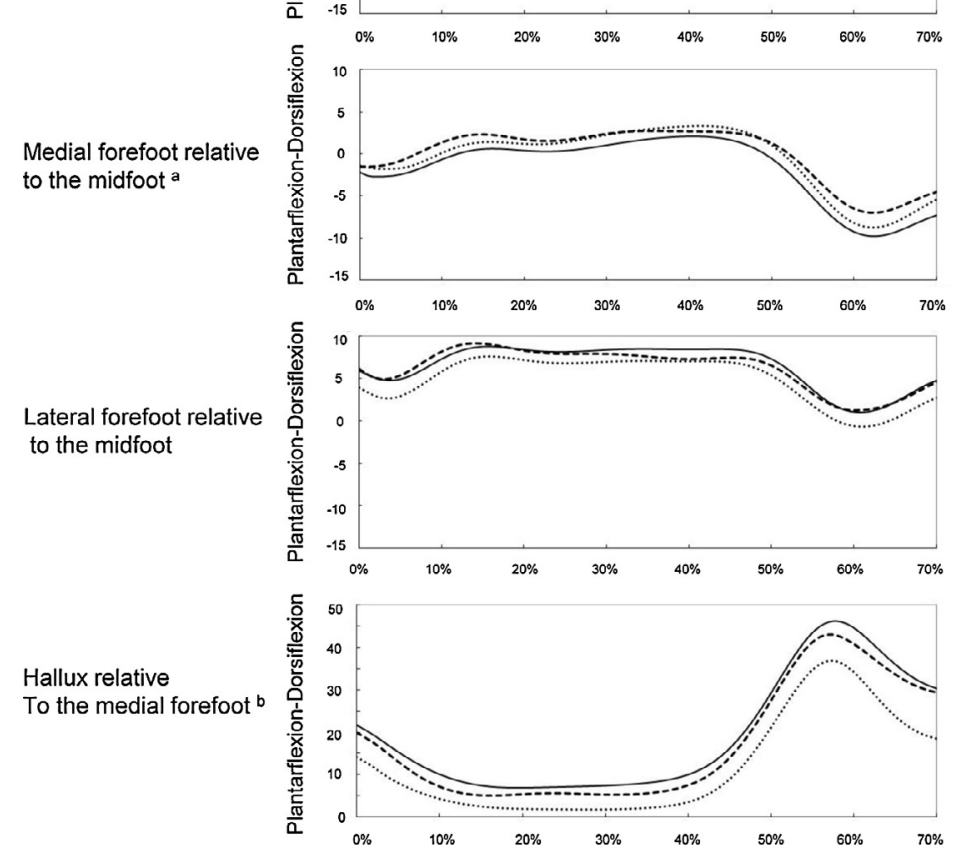
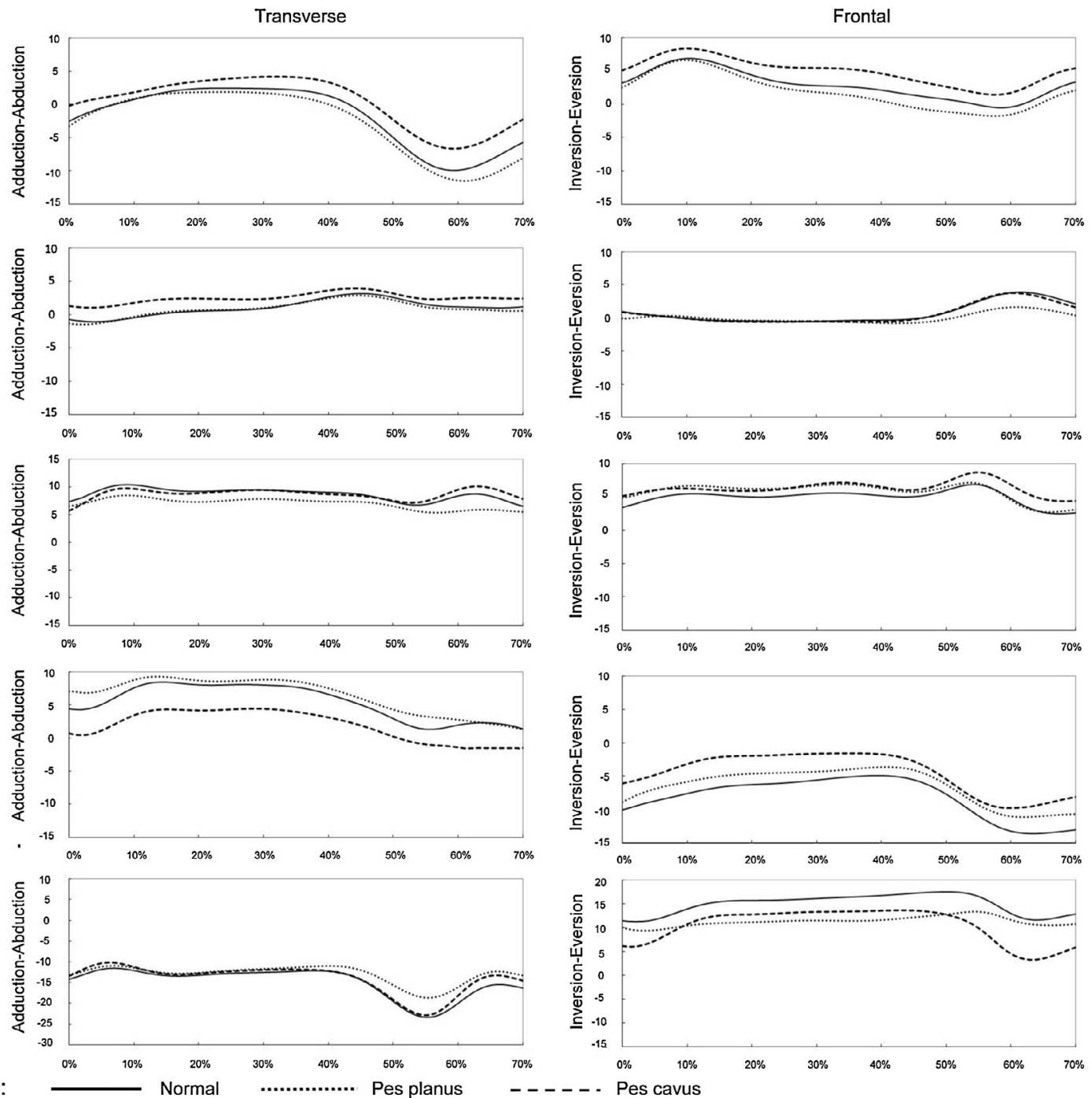
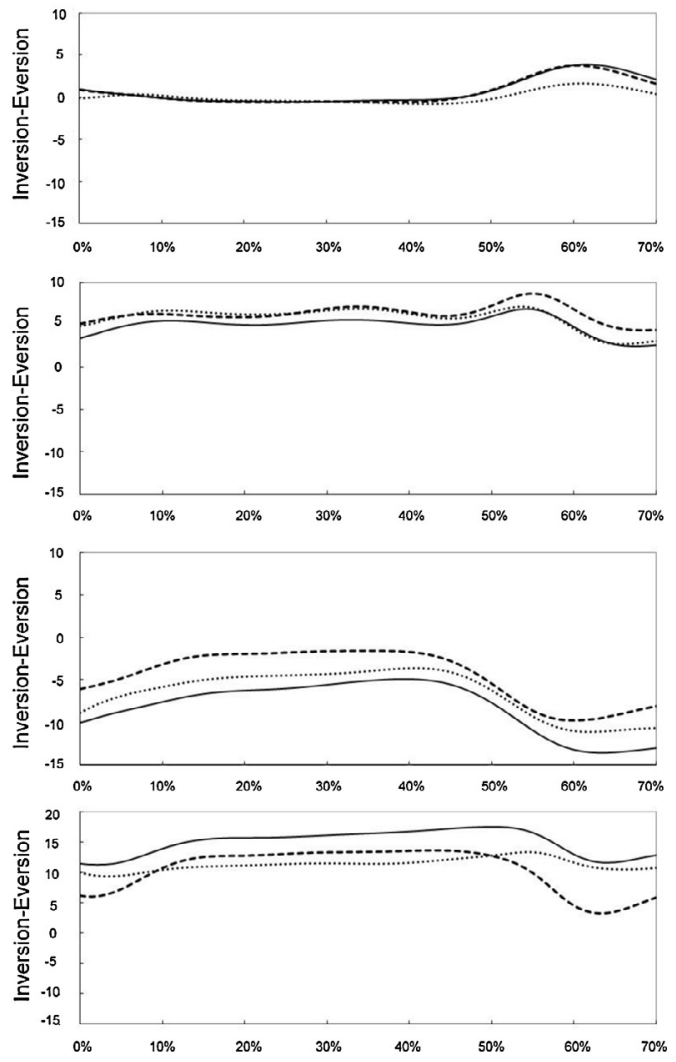

$0 \% \quad 10 \%$
Pes cavus

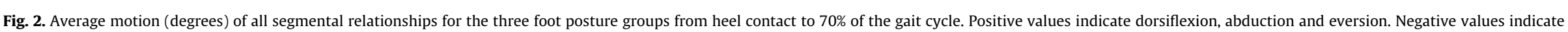

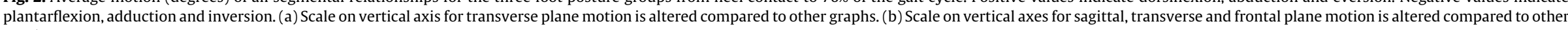
graphs. 


\section{Discussion}

This study provides a novel comparison of foot kinematics between normal, planus and cavus foot posture groups. Further strengths compared to previous work include the use of valid foot posture classification measures based on normative data and the use of a multi-segment foot model that seeks to minimise violation of the rigid body assumption and demonstrates motion of functional foot segments that were identified in invasive bone pin and cadaver research $[11,18]$. The findings indicate that cavus feet have a distinct pattern of motion compared to both normal and planus feet. Three key differences in overall function between the foot posture groups were: (i) altered frontal and transverse plane angles of the rearfoot in the cavus group; (ii) less motion of the midfoot in the sagittal and transverse planes during initial contact and midstance in the cavus group; and (iii) reduced midfoot frontal plane ROM during pre-swing in the planus group.

Before discussing our findings in detail, it is important to consider that all kinematic parameters were calculated relative to the position of the foot in a relaxed standing reference position rather than an examiner-determined 'neutral' reference position. This approach was taken due to the questionable reliability and validity of placing participants in a neutral position, and because this position may not be reached during gait $[19,20]$. Although we believe that this approach is preferable, the use of a relaxed reference position can result in apparently counter-intuitive findings compared to studies that have used a neutral reference position. For example, a planus foot may demonstrate greater rearfoot eversion than a normal foot when a subtalar neutral position reference position is used [21], but such a finding may not be present when using the relaxed reference position approach as the rearfoot is already more everted to begin with in the reference position. Arguably, therefore, the comparison of absolute angular values between foot posture groups is actually a comparison of the extent to which the feet move away from their position during relaxed standing. However, ROM data is independent of this issue and can be directly compared between groups.

The first of three key findings in this study was that the cavus group, compared to the normal and planus groups, demonstrated greater peak eversion and abduction of the rearfoot. There are only two other studies that are suitable for direct comparison, as they used the same reference position and a cavus group [22,23]. Findlow and colleagues [22] used the same multi-segment foot model and found a similar everted and abducted position of the rearfoot for cavus compared to planus feet. Similar findings have also been reported in studies that compared planus with normal foot posture, in which planus feet maintained a less everted position throughout gait $[21,24]$. As cavus feet display only slightly higher peak plantar pressure in the lateral heel compared to planus feet [25], it is possible that the rearfoot positioning found in our study is indicative of a strategy to centralise plantar pressure and reduce force applied to lateral structures of the foot. In contrast, Powell and colleagues [23] reported that planus feet exhibit greater peak eversion compared to cavus feet. However, this difference was due to greater eversion excursion during initial contact rather than during midstance or propulsion as in our study. Other studies that used different reference positions to ours report inconsistent results, with two finding greater peak eversion in pes planus $[3,21]$ compared to three that found no significant differences $[8,26,27]$.

The second key finding was that the midfoot of the cavus group exhibited less peak dorsiflexion and transverse plane ROM during initial contact and midstance. This is indicative of reduced deformation of the medial longitudinal arch, which is thought to contribute to shock attenuation [5]. As increased vertical loading rates and tibial shock (peak positive acceleration) are associated with injuries such as stress fractures [28], this finding could be of clinical importance. However, impact force data measured concurrently are needed to confirm the proposed relationship between medial longitudinal arch deformation and shock attenuation during gait.

The third key finding was that the midfoot of the planus group showed less ROM in the frontal plane, which is associated with reduced eversion of the midfoot on the rearfoot during pre-swing. Two comparative studies that investigated midfoot frontal plane motion found little difference in this variable between foot types $[22,23]$. However, our finding is consistent with biomechanical theory, which suggests that planus feet are comparatively less capable of undertaking the coordinated supinatory motion of the foot that occurs during terminal stance and pre-swing [29]. This supinatory motion, in anatomical terms, has been reported to result in important realignment of the calcaneo-cuboid joint [30]. This, along with tensile forces in plantar tissues due to toe flexion is thought to increase the stiffness of the midfoot and forefoot during the propulsive phase of gait [31]. In planus feet, it is possible that muscles such as tibialis posterior are unable to exert the necessary force to produce eversion of the midfoot on the rearfoot during propulsion. This theory is supported by EMG studies that have reported increased intensity of tibialis posterior muscle activity during propulsion in planus feet [9].

The majority of significant kinematic differences ( 31 out of a total of 36) were found in the cavus group compared to both the normal and planus groups. In real terms, the sizes of these significant differences were small, with all angular variations less than $7.7^{\circ}$. However, the magnitude of effect sizes are classified as medium or large [32] and were typical of those found in other kinematic studies of foot posture, which we summarised in our recent systematic review [10]. It is possible that small angular differences are clinically meaningful in the development of lower limb overuse injury when performing highly repetitive tasks such as jogging and running, although further research is needed to confirm this.

The findings of this study need to be considered in the context of two limitations. Firstly, we examined kinematic data in isolation and hence conclusions about the influence of foot posture on overall biomechanics are difficult without concurrent biomechanical information, such as kinetics and EMG. Secondly, a homogenous cohort of young, healthy participants was recruited, so our findings may not be generalisable to a symptomatic population.

\section{Conclusion}

The findings of this study confirm that different foot postures are associated with differences in movement of the foot when walking. Specifically, the cavus feet displayed less motion during initial contact and midstance, while planus feet exhibited less midfoot ROM during pre-swing.

\section{Authors' contribution}

Study concept and design: AKB, GSM, HBM, KBL. Acquisition of data: AKB, PL, CJN. Analysis and interpretation of data: AKB, PL, GSM, HBM, KBL. Drafting of manuscript: AKB, PL, GSM, HBM, CJN, KBL.

\section{Acknowledgement}

The authors would like to acknowledge and thank Dr. Anthony Schache from the Department of Mechanical Engineering at the University of Melbourne for his work in developing the software code for the foot model that was used in this project. HBM is a National Health and Medical Research Council Senior Research Fellow (ID: 1020925). 
Conflict of interest: The authors declare that there are no conflicts of interest.

\section{Appendix A. Supplementary data}

Supplementary data associated with this article can be found, in the online version, at http://dx.doi.org/10.1016/j.gaitpost. 2015.03.004.

\section{References}

[1] Yates B, White S. The incidence and risk factors in the development of media tibial stress syndrome among naval recruits. Am J Sports Med 2004:32:772 80

[2] Burns J, Keenan A-M, Redmond A. Foot type and overuse injury in triathletes. J Am Podiatr Med Assoc 2005:95:235-41.

[3] Cobb SC, Tis LL, Johnson JT, Wang YT, Geil MD, McCarty FA. The effect of lowmobile foot posture on multi-segment medial foot model gait kinematics. Gait Posture 2009;30:334-9.

[4] Nigg BM, Cole GK, Nachbauer W. Effects of arch height of the foot on angular motion of the lower extremities in running. J Biomech 1993;26:909-16.

[5] Barnes A, Wheat J, Milner C. Association between foot type and tibial stress injuries: a systematic review. Br J Sports Med 2008;42:93-8.

[6] Williams DS, McClay IS, Hamill J, Buchanan TS. Lower extremity kinematic and kinetic differences in runners with high and low arches. J Appl Biomech 2001;17:153-63.

[7] Burns J, Crosbie J, Hunt A, Ouvrier R. The effect of pes cavus on foot pain and plantar pressure. Clin Biomech (Bristol Avon) 2005;20:877-82.

[8] Hunt AE, Smith RM. Mechanics and control of the flat versus normal foot during the stance phase of walking. Clin Biomech (Bristol Avon) 2004:19:391-7.

[9] Murley GS, Menz HB, Landorf KB. Foot posture influences the electromyographic activity of selected lower limb muscles during gait. J Foot Ankle Res 2009;2:35.

[10] Buldt AK, Murley GS, Butterworth P, Levinger P, Menz HB, Landorf KB. The relationship between foot posture and lower limb kinematics during walking: A systematic review. Gait Posture 2013;38:363-72.

[11] Nester CJ, Liu AM, Ward E, Howard D, Cocheba J, Derrick T. Error in the description of foot kinematics due to violation of rigid body assumptions. J Biomech 2010;43:666-72.

[12] Redmond AC, Crane YZ, Menz HB. Normative values for the Foot Posture Index. J Foot Ankle Res 2008;1:6.

[13] Cavanagh PR, Rodgers MM. The arch index: a useful measure from footprints. Biomech 1987;20:547-51.
[14] Cowan DN, Jones BH, Robinson JR. Foot morphologic characteristics and risk of exercise-related injury. Arch Fam Med 1993:2:773-7.

[15] Murley GS, Menz HB, Landorf KB. A protocol for classifying normal- and flatarched foot posture for research studies using clinical and radiographic measurements. J Foot Ankle Res 2009;2:22

[16] Nester C, Jones R, Liu A, Howard D, Lundberg A. Arndt A, et al. Foot kinematics during walking measured using bone and surface mounted markers. J Biomech 2007; 40:3412-23.

[17] Tabachnick BG, Fidell LS, Osterlind SJ. Using multivariate statistics. In: Needham heights. MA: Allyn \& Bacon; 2001.

[18] Lundgren P, Nester C, Liu A, Arndt A, Jones R, Stacoff A, et al. Invasive in vivo measurement of rear-, mid- and forefoot motion during walking. Gait Posture 2008;28:93-100.

[19] McPoil TG, Hunt GC. Evaluation and management of foot and ankle disorders: present problems and future directions. J Orthop Sports Phys Ther 1995;21:381-8.

[20] Menz HB. Clinical hindfoot measurement: a critical review of the literature. Foot 1995;5:57-64.

[21] Houck JR, Tome JM, Nawoczenski DA. Subtalar neutral position as an offset for a kinematic model of the foot during walking. Gait Posture 2008;28:29-37.

[22] Findlow AH, Nester CJ, Bowker P. Foot kinematics in patients with two patterns of pathological plantar hyperkeratosis. J Foot Ankle Res 2011;4:7.

[23] Powell DW, Long B, Milner CE, Zhang S. Frontal plane multi-segment foot kinematics in high- and low-arched females during dynamic loading tasks. Hum Mov Sci 2011;30:105-14.

[24] Hunt AE, Smith RM, Torode M. Extrinsic muscle activity, foot motion and ankle joint moments during the stance phase of walking. Foot Ankle Int $2001 ; 22: 31-41$

[25] Hillstrom HJ, Song J, Kraszewski AP, Hafer JF, Mootanah R, Dufour AB, et al. Foot type biomechanics part 1: structure and function of the asymptomatic foot. Gait Posture 2013;37:445-51.

[26] Levinger P, Murley GS, Barton CJ, Cotchett MP, McSweeney SR, Menz HB. A comparison of foot kinematics in people with normal- and flat-arched feet using the Oxford foot model. Gait Posture 2010;32:519-23.

[27] Levinger P, Menz HB, Fotoohabadi MR, Feller JA, Bartlett JR, Bergman NR. Foot posture in people with medial compartment knee osteoarthritis. J Foot Ankle Res 2010;3:29.

[28] Milner CE, Ferber R, Pollard CD, Hamill J, Davis IS. Biomechanical factors associated with tibial stress fracture in female runners. Med Sci Sports Exerc 2006;38:323-8.

[29] Elftman H. The transverse tarsal joint and its control. Clin Orthop 1960; $16: 41-6$

[30] Bojsen-Møller F. Calcaneocuboid joint and stability of the longitudinal arch of the foot at high and low gear push off. J Anat 1979;129:165-76.

[31] Fuller EA. The windlass mechanism of the foot. A mechanical model to explain pathology. J Am Podiatr Med Assoc 2000;90:35-46

[32] Hopkins WG. A new view of statistics, http://newstatsi.org [accessed 30.01.15]. 